\title{
Study of Different Qualitative Traits in Dolichos Bean (Dolichos lablab L., Var. typicus Prain) Germplasm
}

\author{
K. Jyothi Reddy ${ }^{1 *}$, B. Neeraja Prabhakar ${ }^{2}$, P. Saidaiah ${ }^{3}$ and S.R. Pandravada ${ }^{4}$ \\ ${ }^{1}$ College of Horticulture, Sri Konda Laxman Telangana State Horticultural University, \\ Rajendranagar, Hyderabad-500030, Telangana, India \\ ${ }^{2}$ Department of Horticulture, College of Agriculture, Professor Jayashankar Telangana State \\ Agricultural University, Rajendranagar, Hyderabad-500030, Telangana, India \\ ${ }^{3}$ Department of Genetics and Plant Breeding, College of Horticulture, SKLTSHU, \\ Rajendranagar, Hyderabad-500030, Telangana, India \\ ${ }^{4}$ Economic Botany, NBPGR Regional Station, Rajendranagar, Hyderabad-500030, \\ Telangana, India \\ *Corresponding author
}

\section{A B S T R A C T}

Thirty five genotypes of dolichos bean germplasm lines were evaluated for different

\section{Keywords}

Dolichos bean (Dolichos lablab L. var. typicus Prain), Phaseolinae

Article Info

Accepted: 04 September 2018 Available Online: 10 October 2018 qualitative traits at Vegetable Research Station, Agriculture Research Institute, Rajendranagar, Hyderabad during August, 2016 to March, 2017. Quality traits revealed that there is a considerable variability in dolichos bean germplasm for most of the traits like stem colour (green, dark green and purple), leaf vein colour (light green and green, purple), leaf density (sparse and intermediate), flower colour (purple, white and dark purple), growth habit (pole and bush), pod shape (straight, curved and intermediate), pod colour dark purple in IC-446584, pod beak (long, short and medium), pod suture (green, dark green and purple), pod curvature (straight and curved), pod surface (smooth and wrinkled), seed colour (black, brown and cream) and seed shape (flat, round and oblong). Whereas there was no variability observed in traits like leaf vein colour, pod suture and pod beak colour. These characters can be utilized as morphological markers in selections and breeding programmes.

\section{Introduction}

Dolichos bean (Dolichos lablab L. var. typicus Prain) $(2 \mathrm{n}=2 \mathrm{x}=22)$ belongs to family Leguminaceae sub-family Faboideae, tribe Phaseoleae and sub-tribe Phaseolinae which is commonly known as Indian bean, Hyacinth bean, sem, Egyptian kidney bean, bonavist bean, avarai and avari chikkudu is one of the most popular traditional vegetables extensively cultivated in India. The pods are naturally rich in carbohydrates, proteins, fat and fibers, as well as minerals which include $\mathrm{Ca}, \mathrm{P}$ and $\mathrm{Fe}$ (Naeem et al., 2009).

The protein content of pods and seeds ranges from $10-19 \%$ and $15-25 \%$ respectively. Among the legumes, dolichos bean 
constituents an important source of therapeutic agents used in the modern as well as traditional system of medicine (Morris, 2009). In fact it is considered as a multipurpose crop since it is used for forage, soil improvement, soil protection and weed control (Mass, 2005).

\section{Materials and Methods}

Thirty five dolichos bean germplasm lines along with the three checks were grown in Randomized Block Design with two replications.

Thirty five genotypes viz., IC-261010, IC383197, IC-384066, IC-413709, IC-413710, IC-424813, IC-426988, IC-427424, IC427428, IC-427436, IC-427462, IC-446571, IC-446573, IC-446581, IC-446583, IC446584, IC-446591, IC-546349, IC-546376, IC-546387, IC-546388, IC-565181, IC598467, NSB-2010/029, NSJ/NAIP/192, PSR13183, PSRJ-13039, PSRJ-13114-2, RJR-03, RJR-387, SGD136, SNJ-11-068, RND-1 (check), Arka Jay(check) and Arka Vijay (check)

The data on qualitative characters were recorded as per minimal descriptors of NBPGR (Srivastava et al., 2001). Qualitative data on 13 traits were recorded on ten randomly selected plants in each genotype and the details of trait, classification and stage of scoring are presented in Table 1.

\section{Results and Discussion}

Thirty five genotypes of dolichos bean germplasm under present investigation were characterized based on 13 qualitative traits (Table 2) and (Plate 1.1 and 1.2). The present observations are similar with the findings of Chattopadhyay and Dutta (2010) and Chaitanya et al., (2014). Stem colour revealed that one genotype exhibited dark green stem colour, four genotypes showed purple stem colour and 30 genotypes showed green stem colour. Leaf vein colour of 28 genotypes exhibited light green in colour, five genotypes showed purple colour and 2 genotypes are green in colour. Leaf density of 30 genotypes was found to be intermediate and the remaining five genotypes showed sparse leaf density. Plant growth habit revealed that 33 genotypes were pole type plant growth habit and remaining two genotypes were found to be bush type plant growth habit.

Flower colour revealed that 25 genotypes exhibited white flower colour, nine genotypes showed purple flower colour and one genotype showed dark purple flower colour. Pod colour of 25 genotypes was found to be green in colour, three genotypes showed dark green in colour, five genotypes showed light green in colour, one genotype showed purple colour and remaining one genotype showed dark purple colour. Pod shape of six genotypes was straight, two genotypes were curved and remaining 27 genotypes were intermediate with respect to it.

Pod surface of 28 genotypes had smooth pod surface and remaining seven genotypes had wrinkled pod surfaces. Pod curvature of thirty genotypes showed curved pod curvature and remaining five genotypes showed straight pod curvature. Pod beak revealed that 19 genotypes were with short pod beak, 13 genotypes had medium pod beaks and remaining three genotypes with long pod beaks. Pod suture colour of thirty genotypes showed green colour pod suture and remaining five genotypes showed cream colour pod suture. Seed colour of sixteen genotypes seeds were found to be brown in colour, eight genotypes showed black colour seeds and remaining 11 genotypes seeds were cream in colour. Seed shape of 25 genotypes was found oblong, five genotypes were round in shape and remaining five genotypes were flat in shape. 
Plate.1 Variations in pods of 35 genotypes of dolichos bean including checks

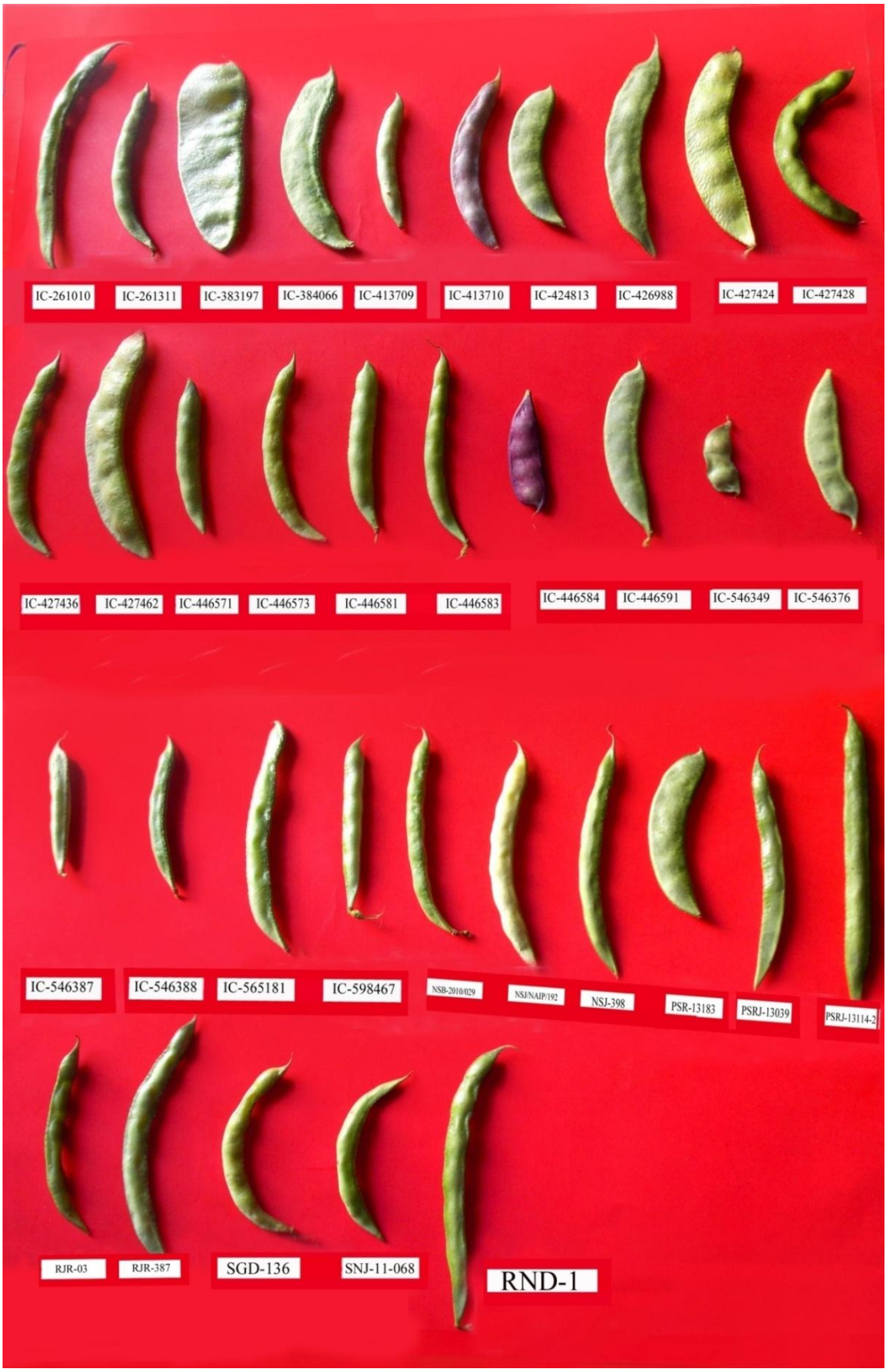


Plate. 2 Variations in seeds of 35 genotypes of dolichos bean including checks
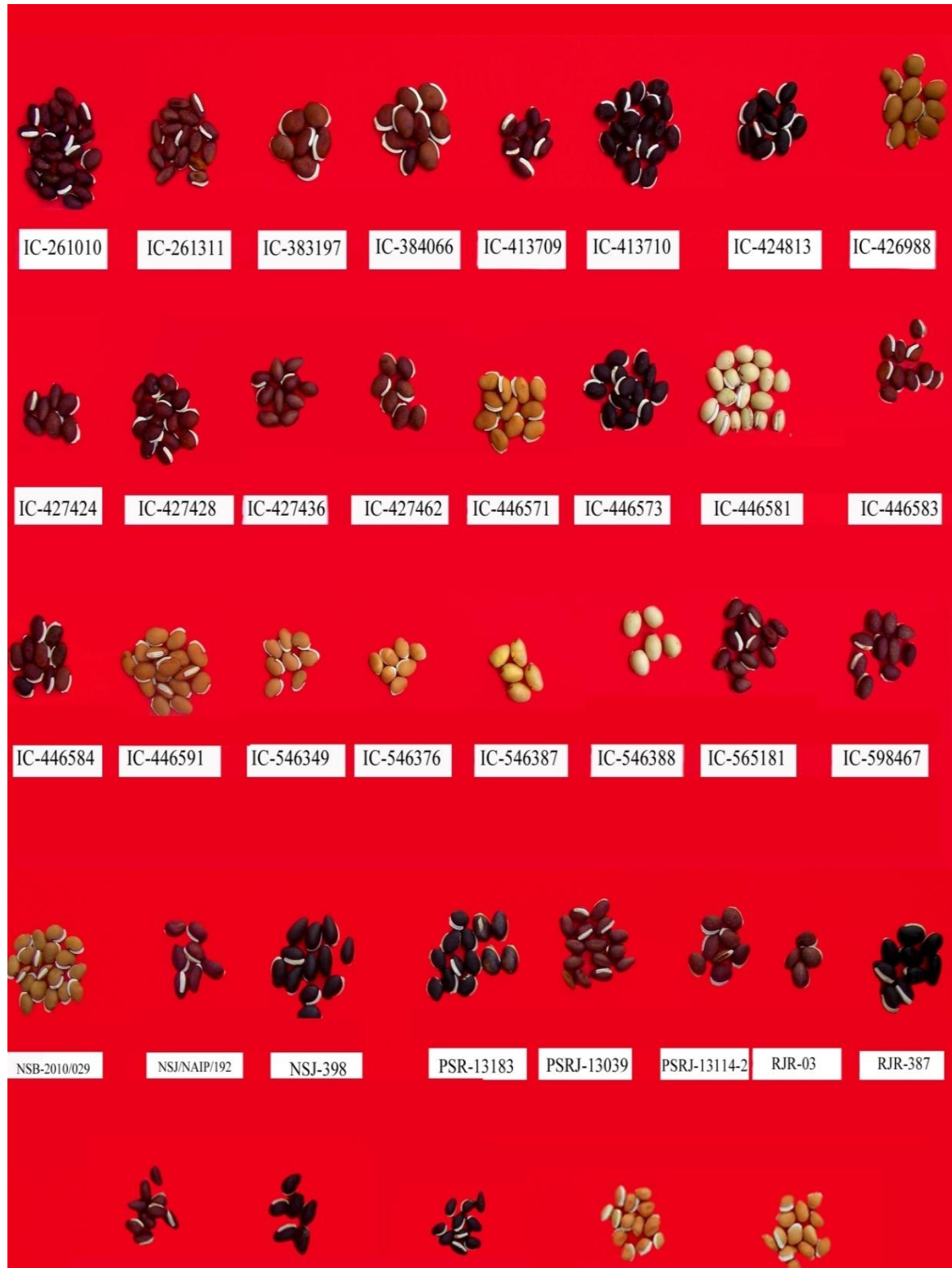

$\begin{array}{llllll}\text { SGD-136 } & \text { SNJ-11-068 } & \text { RND-1 } & \text { ARKA JAY } & \text { ARKA VIJAY }\end{array}$ 
Table.1 Classification and stage of scoring of 13 qualitative traits in dolichos bean

\begin{tabular}{|c|c|c|c|}
\hline S. No. & Qualitative trait & Classification & Stage of scoring \\
\hline 1. & Plant growth habit & Bush, Semi pole and Pole & At flowering stage \\
\hline 2. & Leaf vein colour & Light Green, Green and Purple & $\begin{array}{l}\text { Fully developed primary } \\
\text { leaves on inner surface }\end{array}$ \\
\hline 3. & Leaf density & Sparse, Intermediate and Dense & Vegetative growth stage \\
\hline 4. & Stem colour & $\begin{array}{l}\text { White, Light green, Green, Dark green, } \\
\text { Purple, Dark purple and Greenish } \\
\text { purple }\end{array}$ & Vegetative growth stage \\
\hline 5. & Flower colour & $\begin{array}{l}\text { White, Cream, Purple, Dark purple and } \\
\text { Blue }\end{array}$ & $\begin{array}{l}\text { Fully developed flower } \\
\text { bud before it start anthesis }\end{array}$ \\
\hline 6. & Pod shape & Straight, Intermediate and Curved & Fresh matured pod \\
\hline$\overline{7 .}$ & Pod colour & $\begin{array}{l}\text { White, Cream, Light green, Green Dark } \\
\text { green, Light purple, Purple and Dark } \\
\text { purple }\end{array}$ & Fresh matured pod \\
\hline$\overline{8 .}$ & Pod beak & Short, Medium and Long & Fresh matured pod \\
\hline 9. & Pod curvature & Straight, Curved and Highly curved & Fresh matured pod \\
\hline 10. & Pod suture colour & White, Cream, Green and Purple & Fresh matured pod \\
\hline 11. & Pod surface & Smooth and Wrinkled & Fresh matured pod \\
\hline 12. & Seed colour & $\begin{array}{l}\text { Black, Brown, Cream, Yellow, Purple, } \\
\text { Brick red, Brown yellow and Mottled }\end{array}$ & Dry matured pod \\
\hline 13. & Seed shape & $\begin{array}{l}\text { Cylindrical, Round, Flat, Square, } \\
\text { Oblong and Elliptical }\end{array}$ & Dry matured pod \\
\hline
\end{tabular}


Table.2 Qualitative traits of 35 genotypes of dolichos bean

\begin{tabular}{|c|c|c|c|c|c|c|c|c|c|c|c|c|c|}
\hline $\begin{array}{c}\text { ACCESSION } \\
\text { NO }\end{array}$ & Stem colour & $\begin{array}{l}\text { Leaf vein } \\
\text { color }\end{array}$ & Leaf density & $\begin{array}{l}\text { Plant } \\
\text { growth } \\
\text { habit }\end{array}$ & Flower colour & Pod colour & Pod shape & Pod surface & $\begin{array}{l}\text { Pod } \\
\text { curvature }\end{array}$ & Pod beak & $\begin{array}{l}\text { Pod suture } \\
\text { colour }\end{array}$ & $\begin{array}{l}\text { Seed } \\
\text { colour }\end{array}$ & $\begin{array}{l}\text { Seed } \\
\text { shape }\end{array}$ \\
\hline IC-261010 & Green & Light green & Intermediate & Pole & Purple & Dark green & straight & Smooth & straight & Long & Green & Black & Oblong \\
\hline IC-383197 & Green & Light green & Intermediate & Pole & White & Green & Curved & Wrinkled & Curved & Short & Green & Brown & Flat \\
\hline IC-384066 & Green & Light green & Intermediate & Pole & White & Green & Curved & Wrinkled & Curved & Short & Green & Brown & Flat \\
\hline IC-413709 & Green & Light green & Intermediate & Pole & White & Light green & Intermediate & Smooth & Curved & Long & Cream & Black & Oblong \\
\hline IC-413710 & Purple & Purple & Intermediate & Pole & Dark purple & Purple & Intermediate & Smooth & Curved & Medium & Cream & Black & Flat \\
\hline IC-424813 & Green & Light green & Intermediate & Pole & Purple & Green & Intermediate & Smooth & Curved & Short & Green & Black & Oblong \\
\hline IC-426988 & Green & Light green & Sparse & Pole & White & Green & Intermediate & Wrinkled & Curved & Medium & Green & Cream & Oblong \\
\hline IC-427424 & Green & Light green & Intermediate & Pole & White & Green & Straight & Wrinkled & Curved & Short & Green & Brown & Oblong \\
\hline IC-427428 & Green & Light green & Intermediate & Pole & White & Dark green & Intermediate & Smooth & Curved & Short & Green & Brown & Oblong \\
\hline IC-427436 & Green & Light green & Intermediate & Pole & White & Green & Straight & Wrinkled & Straight & Medium & Green & Brown & Oblong \\
\hline IC-427462 & Green & Light green & Intermediate & Pole & White & Light green & Intermediate & Wrinkled & Curved & Short & Green & Brown & Round \\
\hline IC-446571 & Green & Light green & Intermediate & Pole & White & Green & Intermediate & Smooth & Curved & Short & Cream & Cream & Flat \\
\hline IC-446573 & Green & Purple & Intermediate & Pole & Purple & Green & Intermediate & Smooth & Curved & Medium & Green & Black & Round \\
\hline IC-446581 & Green & Light green & Intermediate & Pole & White & Light green & Straight & Smooth & Straight & Medium & Green & Cream & Round \\
\hline IC-446583 & Green & Light green & Sparse & Pole & White & Green & Straight & Smooth & Straight & Medium & Green & Brown & Oblong \\
\hline IC-446584 & Purple & Purple & Intermediate & Pole & Purple & Dark purple & Intermediate & Smooth & Curved & Short & Cream & Brown & Oblong \\
\hline IC-446591 & Green & Light green & Intermediate & Pole & White & Green & Intermediate & Smooth & Curved & Short & Green & Cream & Round \\
\hline IC-546349 & Green & Light green & Sparse & Pole & White & Green & Intermediate & Smooth & Curved & Medium & Green & Cream & Oblong \\
\hline IC-546376 & Green & Light green & Intermediate & Pole & White & Green & Intermediate & Smooth & Curved & Short & Green & Cream & Oblong \\
\hline IC-546387 & Green & Light green & Intermediate & Pole & White & Light green & Intermediate & Smooth & Curved & Short & Cream & Cream & Round \\
\hline IC-546388 & Green & Light green & Intermediate & Pole & White & Green & Intermediate & Smooth & Curved & Short & Green & Cream & Oblong \\
\hline IC-565181 & Green & Light green & Intermediate & Pole & White & Green & Intermediate & Smooth & Curved & Short & Green & Brown & Oblong \\
\hline IC-598467 & Green & Light green & Intermediate & Pole & White & Green & Intermediate & Smooth & Curved & Medium & Green & Brown & Oblong \\
\hline NSB-2010/029 & Green & Light green & Intermediate & Pole & White & Green & Intermediate & Smooth & Curved & Short & Green & Cream & Oblong \\
\hline NSJ/NAIP/192 & Green & Light green & Intermediate & Pole & White & Light green & Intermediate & Smooth & Curved & Short & Cream & Brown & Oblong \\
\hline $\begin{array}{c}\text { ACCESSION } \\
\text { NO }\end{array}$ & Stem colour & $\begin{array}{l}\text { Leaf vein } \\
\text { colour }\end{array}$ & Leaf density & $\begin{array}{l}\text { Plant } \\
\text { growth } \\
\text { habit }\end{array}$ & Flower colour & Pod colour & Pod shape & Pod surface & $\begin{array}{l}\text { Pod } \\
\text { curvature }\end{array}$ & Pod beak & $\begin{array}{l}\text { Pod suture } \\
\text { colour }\end{array}$ & $\begin{array}{l}\text { Seed } \\
\text { color }\end{array}$ & $\begin{array}{l}\text { Seed } \\
\text { shape }\end{array}$ \\
\hline PSR-13183 & Purple & Purple & Intermediate & Pole & Purple & Green & Intermediate & Wrinkled & Curved & Medium & Green & Black & Oblong \\
\hline PSRJ-13039 & Green & Light green & Intermediate & Pole & White & Dark green & Intermediate & Smooth & Curved & Medium & Green & Brown & Flat \\
\hline PSRJ-13114-2 & Green & Light green & Intermediate & Pole & White & Green & Intermediate & Smooth & Curved & Medium & Green & Brown & Oblong \\
\hline RJR-03 & Green & Light green & Intermediate & Pole & White & Green & Intermediate & Smooth & Curved & Short & Green & Brown & Oblong \\
\hline RJR-387 & Purple & Purple & Intermediate & Pole & Purple & Green & Intermediate & Smooth & Curved & Medium & Green & Black & Oblong \\
\hline SGD-136 & Green & Light green & Intermediate & Pole & White & Green & Straight & smooth & Straight & Short & Green & Brown & Oblong \\
\hline SNJ-11-068 & Dark green & Green & Intermediate & Pole & Purple & Green & Intermediate & Smooth & Curved & Long & Green & Brown & Oblong \\
\hline RND-01@ & Green & Green & Intermediate & Pole & Purple & Green & Intermediate & Smooth & Curved & medium & Green & Black & Oblong \\
\hline ARKA JAY ( & Green & Light green & Sparse & Bush & Purple & Green & Intermediate & Smooth & Curved & Short & Green & Cream & Oblong \\
\hline $\begin{array}{l}\text { ARKA } \\
\text { VIJAY@ }\end{array}$ & Green & Light green & Sparse & Bush & White & Green & intermediate & Smooth & Curved & Short & Green & Cream & Oblong \\
\hline
\end{tabular}


Quality traits revealed that there is a considerable variability in dolichos bean germplasm for most of the traits like stem colour (green, dark green and purple), leaf vein colour (light green and green, purple), leaf density (sparse and intermediate), flower colour (purple, white and dark purple), growth habit (pole and bush), pod shape (straight, curved and intermediate), pod colour (green in IC-383197, IC-384066, IC424813, IC-426988, IC-427424, IC-427436, IC-446571, IC-446573, IC-446583, IC446591, IC-546349, IC-546376, IC-546388, IC-565181, IC-598467, NSB-2010/029, PSR13183， PSRJ-13114-2， RJR-03， RJR-387, SGD-136, SNJ-11-068, RND-01, ARKA JAY and ARKA VIJAY); light green in IC413709, IC-427462, IC-446581, IC-546387 and NSJ/NAIP/192, dark green in IC-261010, IC-427428 and PSRJ-13039, purple in IC413710, dark purple in IC-446584, pod beak (long, short and medium), pod suture (green, dark green and purple), pod curvature (straight and curved), pod surface (smooth and wrinkled), seed colour (black, brown and cream) and seed shape (flat, round and oblong). Whereas there was no variability observed in traits like leaf vein colour, pod suture and pod beak colour. Dolichos bean genotypes exhibited high variability for all traits like plant growth characters, leaf characters, flower characters, pod characters, seed characters and quality characters.

\section{References}

Chaitanya, V, Reddy, R.V.S.K, Pandravada, S.R, Sujatha, M and Kumar, A.P. 2014.
Correlation and Path Coefficient Analysis in dolichos bean (Dolichos lablab L. var. typicus prain) genotypes Plant Archives Vol. 14 No.1, pp.537540

Chattopadyay, A and Dutta, S. 2010. Characterization and identification of selection indices of pole type dolichos bean. Vegetable crops research bulletin 73:33-45.

Mass, B. L. 2005. Changes in seed morphology, dormancy and germination from wild to cultivated hyacinth bean germplasm (Lablab purpureus L. Papilinoideae.) Genetic Resources and Crop Evaluation: 1-9.

Morris, J.B. 2009. Morphological and reproductive characterization in hyacinth bean (Lablab purpureus (L.) Sweet) gemplasm with clinically proven nutracuetical and pharmaceutical traits for use as a medicinal food. Journal of Dietary Supplements 6(3): 263-279.

Naeem, M, Khan, M. M. A and Siddiqui, M. H. 2009. Triacontanol stimulates nitrogen fixation, enzyme activities, photosynthesis, crop productivity and quality of hyacinth bean (Lablab purpureus L.). Scientia Horticulture 121(4): 389-396.

Srivastava, U, Mahajan, R. K, Gangopadhyay, K. K., Singh, M and Dhilon, B. S. 2001. Minimal Descriptors of AgriHorticultural crops, Vegetable crops part-II.

\section{How to cite this article:}

Jyothi Reddy, K., B. Neeraja Prabhakar, P. Saidaiah and Pandravada, S.R. 2018. Study of Different Qualitative Traits in Dolichos Bean (Dolichos lablab L., Var. typicus Prain) Germplasm. Int.J.Curr.Microbiol.App.Sci. 7(10): 358-364. doi: https://doi.org/10.20546/ijcmas.2018.710.038 